\title{
AVALIAÇÃO DAS HABILIDADES FUNCIONAIS E DE AUTO CUIDADO DE INDIVÍDUOS COM SÍNDROME DE DOWN PERTENCENTES A UMA OFICINA TERAPÊUTICA
}

\section{Evaluation of functional skills and self care of Down's syndrome people included in therapeutic workshop}

\author{
Marielza Regina Ismael Martins (1), Maria Amélia Branco Fecuri (2), \\ Marta Alves Arroyo ${ }^{(3)}$, Márcia Taves Parisi ${ }^{(4)}$
}

\begin{abstract}
RESUMO
Objetivo: avaliar as habilidades funcionais e de auto cuidado de indivíduos com Síndrome de Down em uma oficina interdisciplinar terapêutica. Método: investigação prospectiva, exploratória, comparativa, de corte transversal e abordagem quantitativa com amostra de um grupo teste(T), com síndrome de Down $(\mathrm{N}=17)$ e, grupo controle $(C)$ sem a síndrome $(n=20)$. O instrumento utilizado foi o Inventario de avaliação pediátrica (PEDI) que avalia aspectos funcionais do desempenho de crianças. Resultados: a amostra foi pareada (idade média 5 a $71 \frac{1}{2}$ anos,desvio padrão 1a 6 meses). $O$ grupo T apresentou um repertório de habilidades funcionais na área de autocuidado inferior aos participantes do grupo $C(p<0,05)$. Nas áreas de mobilidade e função social apesar de apresentarem escores menores não houve diferença estatística entre os grupos. Conclusão: a monitoração contínua em atividades coletivas no formato de oficinas pode ser útil no conhecimento sobre o impacto destas atividades no perfil funcional destas crianças.
\end{abstract}

DESCRITORES: Síndrome de Down; Aptidão; Prática de Grupo

\section{INTRODUÇÃO}

A síndrome de Down (SD) é uma condição genética, reconhecida há mais de um século que se caracteriza pela existência de um cromossomo extra ou parte de um cromossomo extra, causando uma triplicação ao invés da duplicação do material genético referente ao par cromossômico $21^{1}$.

(1) Terapeuta Ocupacional; Doutora em Ciências da Saúde; pertencente ao Projeto Ding Down do Hospital de Base e Faculdade de Medicina de São José do Rio Preto/ FAMERP.

(2) Fonoaudióloga; Mestre em Ciências da Saúde; pertencente ao Projeto Ding Down do Hospital de Base e Faculdade de Medicina de São José do Rio Preto/FAMERP.

(3) Fonoaudióloga; Doutora em Ciências da Saúde; pertencente ao Projeto Ding Down do Hospital de Base e Faculdade de Medicina de São José do Rio Preto/FAMERP

(4) Fisioterapeuta; pertencente ao Projeto Ding Down do Hospital de Base e Faculdade de Medicina de São José do Rio Preto/FAMERP.

Conflito de interesses: inexistente
Sua incidência em nascidos vivos é de 1 para cada 600/800 nascimentos, tendo uma média de 8.000 novos casos por ano no Brasil. De acordo com os dados levantados pelo IBGE, com base no Censo de 2000, existem 300 mil pessoas com SD no país, com expectativa de vida de 50 anos, sendo esses dados bastante semelhantes às estatísticas mundiais ${ }^{2,3}$

As respostas motoras, verbais e gráficas da criança com SD são mais lentas devido as suas limitações pelos aspectos internos (déficits na memória de curto prazo, hipoplasia cerebelar, motivação, processos interativos) e externos (ambiente estimulante, envolvimento parental) que tem a possibilidade de ser minimizadas com o apoio do meio ${ }^{4}$.

Estudos atuais ${ }^{4,5}$ referem que o desempenho de pessoas que apresentam SD é mais lento e variável do que de pessoas que não apresentam a síndrome e isto pode ser interpretado como o modo com que as pessoas com SD realizam a reação adaptativa que envolve a forma como este individuo se ajusta 
às demandas para a ação intencional frente a diferentes situações .

Diante deste contexto busca-se oferecer um ambiente solicitador, que promova autonomia e diferentes possibilidades de descobertas de seu potencial, como as oficinas. Oficinas terapêuticas são espaços onde se realizam atividades grupais de socialização, expressão e inserção social ${ }^{6,7}$.

Assim o objetivo é avaliar as habilidades funcionais e de auto cuidado de indivíduos com Síndrome de Down em uma oficina interdisciplinar terapêutica, espaço esse criado para propiciar a experimentação constante para realização de atividades diversas (jogos educativos, atividades manuais, dramatizações, oficina de sucata e recursos expressivos) com a mediatização dos profissionais de saúde envolvidos (fonoaudiologia, terapia ocupacional e fisioterapia). Neste contexto oportunizam-se a promoção de interação com aumento da autonomia, o desenvolvimento do potencial criativo e a valorização da singularidade.

\section{MÉTODO}

Trata-se de uma investigação prospectiva, exploratória, comparativa, de corte transversal com abordagem quantitativa. Foram incluídos indivíduos de ambos os sexos, alocados em dois grupos - 0 grupo teste (T) e o grupo controle (C). O grupo $\mathrm{T}$ foi composto por 17 crianças que apresentam a Síndrome de Down, sendo oito $(47,0 \%)$ do sexo masculino e nove $(53,0 \%)$ do sexo feminino. A idade das crianças variou de 5 a $71 \frac{1}{2}$ anos, portanto, em torno de 1 ano e 6 meses. $O$ grupo $C$ foi composto por 20 crianças que apresentam desenvolvimento normal típico, sem problemas clínicos, diagnóstico de doença ou distúrbio do desenvolvimento e que não fazem uso sistemático de medicação. As crianças dos dois grupos foram pareadas em relação à idade. As crianças do grupo $\mathrm{C}$ foram recrutadas no Ambulatório de Pediatria e também na mesma Oficina as quais acompanhavam ou eram irmãos das crianças com SD e, assim passaram também a fazer parte da Oficina .

As crianças foram avaliadas pelo teste PEDI 8 (Pediatric Evaluation of Disability Inventory) que é um instrumento padronizado norte-americano que documenta de forma quantitativa a capacidade funcional da criança (habilidades) e a independência para realizar atividades de auto-cuidado, mobilidade e função social. O PEDI é uma avaliação realizada por meio de entrevista com pais ou responsáveis que possam informar sobre o desempenho da criança em casa. Este teste avalia aspectos funcionais do desempenho de crianças com idade entre 6 meses e 7 anos e meio, em três áreas e pode ser usado em crianças com idades cronológicas superiores ao limite indicado, desde que o desempenho funcional das mesmas esteja aquém desse limite. A primeira parte do teste avalia as habilidades ou capacidades funcionais da criança; a segunda parte informa sobre a quantidade de ajuda ou assistência que a criança recebe para desempenhar as atividades funcionais, e a terceira parte documenta as modificações do ambiente necessárias para o desempenho de tarefas funcionais. $\mathrm{O}$ desempenho funcional foi avaliado em três aspectos do desenvolvimento: autocuidado (73 itens), mobilidade (59 itens) e função social (65 itens). Cada item é pontuado com escore 0 (zero) se a criança não é capaz de desempenhar a atividade funcional, ou 1 (um) se a atividade fizer parte do repertório de atividades da criança. Os 73 itens funcionais avaliados na escala de autocuidado foram agrupados nas seguintes tarefas: alimentação (14 itens), higiene pessoal (14 itens), banho (10 itens), vestir (20 itens), uso do toalete (5 itens) e controle esfincteriano (10 itens). Os 59 itens funcionais da escala de mobilidade são agrupados nas seguintes tarefas: transferências (24 itens), locomoção em ambientes internos (13 itens), locomoção em ambientes externos (12 itens) e uso da escada (10 itens). Na escala de função social, os 65 itens ou habilidades funcionais foram agrupados da seguinte forma:compreensãofuncional(15itens), verbalização (10 itens), resolução de problemas (5 itens), brincar (15 itens), auto-informação (5 itens), participação da rotina doméstica/comunidade (10 itens) e noção de autoproteção (5 itens). A pontuação da segunda parte do PEDI é dada em uma escala ordinal que varia de 5 (se a criança é independente no desempenho da tarefa, não necessitando de nenhum tipo de assistência do cuidador para realizar a tarefa) a 0 (se a criança é totalmente dependente do cuidador para realizar a tarefa). Os escores intermediários descrevem quantidades variadas de ajuda fornecida pelo cuidador (supervisão: mínima, moderada ou máxima).

Neste estudo foram utilizadas apenas as três escalas das atividades funcionais da parte I do teste PEDI. As crianças foram avaliadas por pesquisadores previamente treinados na aplicação do teste. Cada entrevista durou cerca de 45 minutos.

As oficinas acontecem 1 vez ao mês com duração de 2 horas onde se realiza jogos, histórias, dramatizações, uso da arte do corpo e plásticas em suas diversas formas. A ferramenta é a atividade lúdica, orientada e supervisionada por uma equipe interdisciplinar, com abordagem holística e integrada (terapeutas ocupacionais, fonoaudiólogos e fisioterapeutas). 
Ambos os grupos foram avaliados em dois momentos ( $1^{\circ}$ e $10^{\circ}$ encontro). A reavaliação utilizou os mesmos critérios e então foram comparados os resultados.

A Oficina faz parte de um Projeto cuja adesão é espontânea. Acontece mensalmente em um espaço institucional amplo e adequado a diversas práticas, onde as crianças podem evoluir livremente.

O projeto de pesquisa foi aprovado pelo Comitê de Ética em Pesquisa da FAMERP (4110/2011) e realizado na Oficina Terapêutica vinculada ao Projeto Ding-Down, Hospital de Base/Faculdade de Medicina de São José do Rio Preto (FUNFARME/ FAMERP).

Os dados deste estudo foram analisados utilizando-se $o$ teste $t$ de Student para grupos independentes. Foram comparadas as médias dos escores obtidos pelas crianças dos diferentes grupos, nas áreas do desempenho funcional (autocuidado, mobilidade e função social). Para cada análise foi considerado nível de significância de $95 \%(p<0,05)$.

\section{RESULTADOS}

Os resultados estatísticos revelaram diferença significativa nas habilidades de auto-cuidado entre os grupos avaliados no $1^{\circ}$ e $10^{\circ}$ encontros $(p=0,048)$.

$\mathrm{Na}$ Tabela 1 observam-se os resultados das comparações entre as médias dos escores dos dois grupos, nas três áreas de desempenho funcional.

Tabela 1 - Medidas descritivas das pontuações obtidas no PEDI aplicados aos grupos T ( $n=17)$ e C $(n=20)$ e resultados do teste da comparação dos grupos

\begin{tabular}{ccccccc}
\hline \multicolumn{7}{c}{ Medidas } \\
\hline Área & Grupo & $\mathbf{1}^{\circ}$ Encontro & Mediana & $\mathbf{1 0}^{\circ}$ Encontro & Média \pm dp & $\mathbf{P}$ \\
\hline \multirow{2}{*}{ Auto cuidado } & $\mathrm{C}$ & 68 & 72 & 75 & $71 \pm 2,2$ & \multirow{2}{*}{$0,048^{*}$} \\
& $\mathrm{~T}$ & 30 & 63 & 68 & $62 \pm 4,5$ & \\
\multirow{2}{*}{ Mobilidade } & $\mathrm{C}$ & 56 & 61 & 66 & $61 \pm 1,8$ & \multirow{2}{*}{0,056} \\
& $\mathrm{~T}$ & 40 & 48 & 50 & $45 \pm 4,5$ & \\
\hline \multirow{2}{*}{ Função Social } & $\mathrm{C}$ & 46 & 62 & 66 & $62 \pm 2,5$ & \multirow{2}{*}{0,082} \\
& $\mathrm{~T}$ & 38 & 54 & 63 & $53 \pm 5,2$ & \\
\hline
\end{tabular}

(*) nível descritivo de significância de 0,05.

As crianças com do grupo $T$ apresentaram desempenho significantemente inferior ao das crianças do grupo $\mathrm{C}$ na habilidade funcional de autocuidado $(p<0,05)$. Contudo a média dos escores da mobilidade e função social foi maior no grupo C, porém não alcançou significância estatística.

Nas figuras abaixo estão relacionados às áreas que foram desenvolvidas nos encontros avaliados.
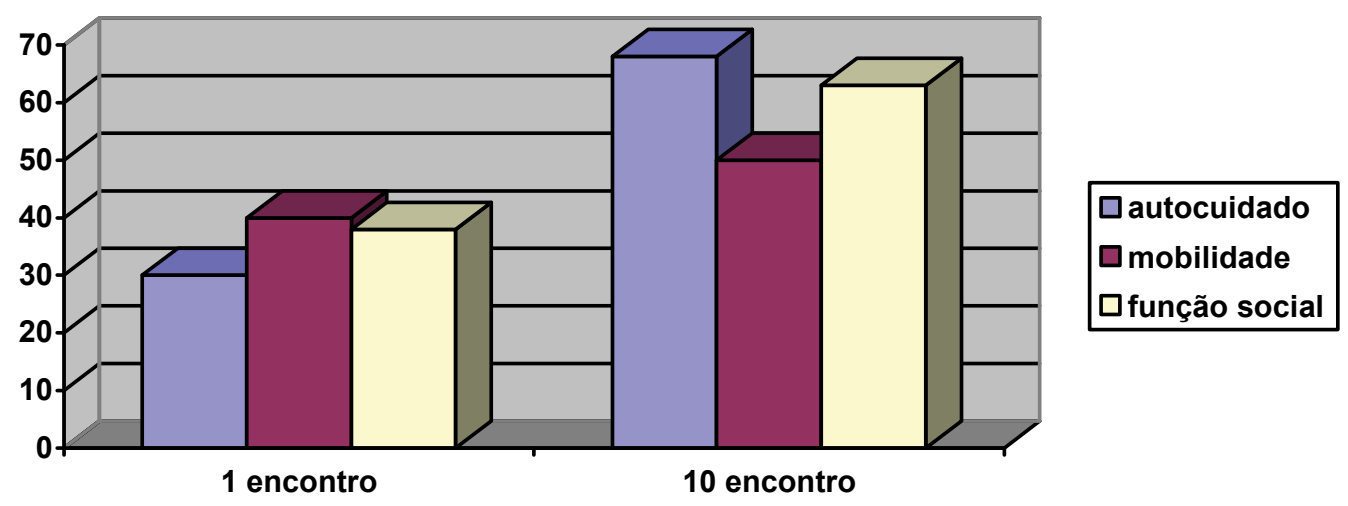

Figura 1 - Áreas avaliadas do grupo $\mathrm{T}(\mathrm{n}=17)$ nos encontros realizados 


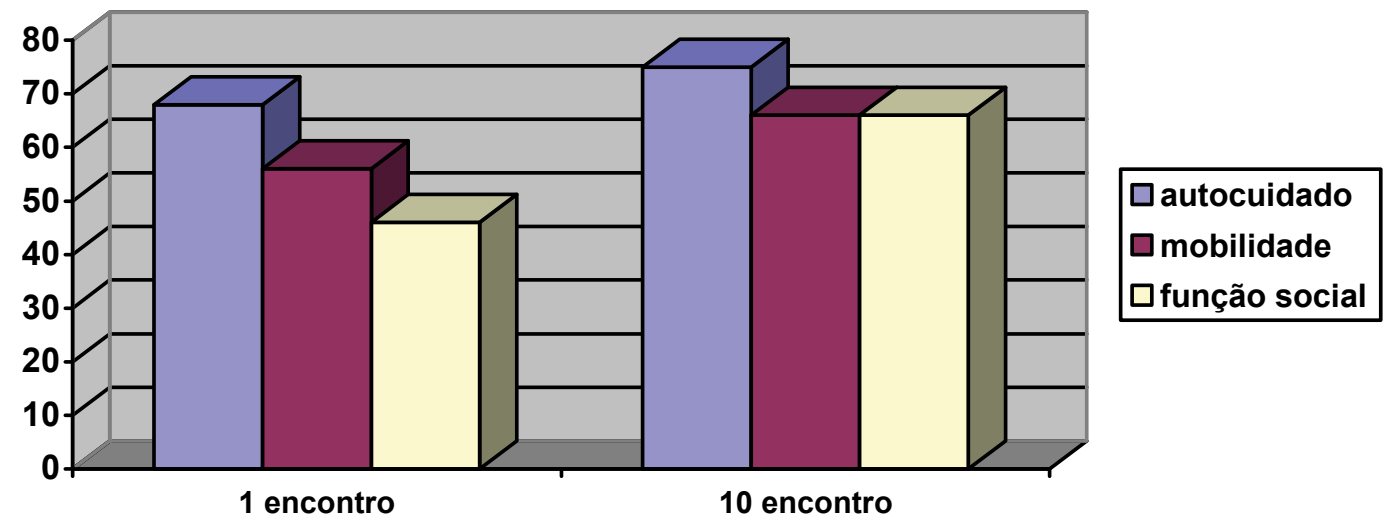

Figura 2 - Áreas avaliadas do grupo C $(n=20)$ nos encontros realizados

\section{DISCUSSÃO}

Neste estudo foram feitas comparações de informações sobre o desempenho funcional através de inventário específico em participantes de uma oficina terapêutica. As idades pareadas permitiram a comparação dos comportamentos funcionais.

Os resultados do estudo sugerem que a promoção do desempenho em tarefas funcionais foi um objetivo alcançado pela oficina. É possível inferir que a promoção desses desempenhos seja decorrente das oficinas, pois esta oportuniza atividades com desafios motores, cognitivos e sociais que os motivam a enfrentar aqueles que eles têm maior dificuldade, contribuindo para que, dessa forma, se desenvolvam física e mentalmente e avancem gradualmente.

O grupo T deste estudo apresentou um repertório de habilidades funcionais na área de autocuidado inferior aos participantes do grupo $\mathrm{C}$, confirmando evidencias existentes na literatura ${ }^{9,10}$, observando que apesar desta diferença, houve aumento do escore ao longo do tempo.

Com relação às áreas de mobilidade e função social o desempenho das crianças do grupo $T$ apesar de inferior comparado ao grupo $\mathrm{C}$ em ambos os momentos, não obtiveram diferença significante. $O$ trabalho de Atkinson e Smith ${ }^{11}$ corrobora estes resultados e mostra que diferentes indivíduos expostos ao mesmo ambiente explanam distintas reações, que serão importantes por toda a vida. Freitas e Rodrigues ${ }^{12}$ relatam que na criança deficiente intelectual o potencial de aprendizagem é caracterizado por graus de incapacidade adaptativa, em que subsiste uma inferioridade mental global. Mas a deficiência também é uma condição na qual o cérebro não necessariamente pode estar impedido de desenvolver, pois não há exposição aos diferentes estímulos interativos que o meio pode oferecer.

No decorrer dos encontros da oficina houve a viabilização de desafios por meio de atividades lúdicas para que o desenvolvimento cognitivo-motor incluindo as habilidades funcionais fosse alcançado. Assim, a interação social foi estimulada de diferentes maneiras de expressão e comunicação.O estímulo permanente das atividades (por meio do manuseio de pincéis, tesouras, lápis, cola, tinta guache, giz de cera.....) e este constante surgimento de obstáculos possibilitaram uma evolução no aspecto motor. Devido à oficina possuir um cunho interdisciplinar ocorreu um estímulo ao desenvolvimento da capacidade cognitiva por meio do vocabulário e da intervenção multisensorial, preconizada na atualidade ${ }^{13}$.

\section{CONCLUSÃO}

No estudo observam-se limitações nas habilidades funcionais de indivíduos com Síndrome de Down participantes de uma oficina quando comparados ao grupo de padrão típico. Estas limitações manifestaram-se significantemente na área de autocuidado, porém nas áreas de mobilidade e função social, apesar de apresentarem um repertório de habilidades mais deficitário, não houve diferença estatística entre os grupos.

Estudos futuros, com monitorização contínua em atividades coletivas no formato de oficinas podem ser úteis no conhecimento sobre o impacto destas atividades no perfil funcional destas crianças. 


\section{ABSTRACT}

Purpose: to evaluate the functional abilities and self-care in Down syndrome people in a therapeutic interdisciplinary workshop. Method: prospective, exploratory, comparative and cross-sectional research and quantitative approach with a sample of a test group $(n=17)$ with Down syndrome and control group $(n=20)$ without the syndrome $(n=20)$. The instrument used was the Pediatric Evaluation of Disability Inventory (PEDI) to evaluate functional aspects of the children's performance. Results: the sample was paired (between 5 and $7 \frac{1}{2}$ years old, standard deviation 1 year and 6 months old). The group $T$ had a repertory of functional abilities in the self-care area lower than the participants in group $C(p<0,05)$. In the mobility and function social areas despite the lower scores, it did not differ between groups. Conclusion: the continuous monitoring of collective activities in the format of workshops can be useful in knowing the impact of such activities in the functional profile of these children.

KEYWORDS: Down Syndrome; Aptitude; Group Practice

\section{REFERÊNCIAS}

1. Dowjat WK, Adayev T, Kuchna I, Nowicki K, Palminiello S, Hwang W, Wegiel J . Trisomy-driven overexpression of DYRK1A kinase in the brain of subjects with Down syndrome. Neurosci. Lett. 2007; 77-81.

2. Schwartzman J.S. O sistema nervoso na síndrome de Down. . In: Schwartzman, J.S. (Org.), Síndrome de Down. São Paulo: Mackenzie 2003; p. 44-81.

3. DATASUS (Departamento de Informática do SUS), 2002. SINASC Sistema de Informação sobre Nascidos Vivos. 1 Agosto 2002 <http://www. datasus.gov.br>

4. Wuang YP, Su CY. Correlations of sensory processing and visual organization ability with participation in school-aged children with Down syndrome.Res Dev Disabil. 2011; 32(6): 2398-407.

5. Heath M, Grierson L, Binsted G, Elliott D. Interhemispheric transmission time in persons with Down syndrome.J Intellect Disabil Res. 2007 Dec;51:972-81.

http://dx.doi.org/10.1590/S1516-18462012005000088

RECEBIDO EM: 21/09/2011

ACEITO EM: 26/01/2012

Endereço para correspondência:

Marielza R. Ismael Martins

Departamento de Ciências Neurológicas

Av. Brigadeiro Faria Lima, 5416 - Vila São Pedro

São José do Rio Preto - SP

CEP: 15090-000

E-mail: marielzamartins@famerp.br
6. Sunelaitis RC, Arruda DC, Marcom SS. A repercussão de um diagnóstico de síndrome de Down no cotidiano familiar: perspectiva da mãe. Acta Paul Enferm. 2007; 20(3): 264-71

7. Pazin AC, Martins MRI. Desempenho funcional de crianças com Síndrome de Down e a qualidade de vida de seus cuidadores.Rev Neurosc. 2007; 15(4):29-33.

8. Haley S M, Coster WJ, Ludlow LH, Haltiwanger J T, Andrellos PJ. Inventário de avaliação pediátrica de disfunção . versão brasileira. Tradução e adaptação cultural: Mancini M C. Belo Horizonte: Laboratórios de Atividade e Desenvolvimento Infantil, Departamento de Terapia Ocupacional, Universidade Federal de Minas Gerais, 2000.

9. Esbensen AJ, Seltzer MM, Krauss MW. Stability and change in health, functional abilities, and behavior problems among adults with and without Down syndrome.Am J Ment Retard. 2008; 113(4):263-77.

10. Volman MJ, Visser JJ, Lensvelt-Mulders GJ. Functional status in 5 to 7 -year-old children with Down syndrome in relation to motor ability and performance mental ability.Disabil Rehabil. 2007; 29(1):25-31.

11. Atkinson RL, Atkinson RC, Smith E. Introdução à Psicologia. Porto Alegre: Artmed, 2002.

12. Freitas DP, Rodrigues GM. O processo de aprendizagem mediado pelo meio líquido: uma experiência com uma pessoa com Síndrome de Momo. Revista Mackenzie de Educação Física e Esporte. 2007;6(3): 119-25.

13. Cowley PM, Ploutz-Snyder LL, Baynard T, Heffernan KS, Young Jae S, Hsu S et al. The effect of progressive resistance training on leg strength, aerobic capacity and functional tasks of daily living in persons with Down syndrome.Disabil Rehabil. 2011 ; 29 (3):98-105. 